\title{
Management of a Production Cell Lubrication System with Model Predictive Control
}

\author{
Andrea Cataldo ${ }^{1}$, Andrea Perizzato ${ }^{2}$, and Riccardo Scattolini ${ }^{2}$ \\ ${ }^{1}$ Institute of Industrial Technology and Automation - National Research Council, \\ Milan, Italy \\ andrea.cataldo@itia.cnr.it \\ ${ }^{2}$ Politecnico di Milano, Dipartimento di Elettronica, \\ Informazione e Bioingegneria, Milan, Italy \\ \{andrea.perizzato, riccardo.scattolini\}@polimi.it
}

\begin{abstract}
The energy efficiency of manufacturing systems represents a topic of huge interest for the management of innovative production plants. In this paper, a production cell based on three operating machines has been taken into account. In particular, each machine has an independent lubrication system whose lubricant is cooled by a centralized cooling system, while the lubrication fluid temperatures must be maintained inside known upper and lower bounds, and the controller of the centralized cooling system has to minimize the cooling power. In order to control the lubrication and cooling processes, a Model Predictive Controller (MPC) has been designed, synthetized, implemented and simulated.

The main advantage of the proposed algorithm consists in the possibility to directly consider the temperature limits together with the maximum bound of the cooling power directly into the optimization problem. This means that the control action is computed using the a-priori knowledge of these bounds, resulting in better temperature profiles then those obtained with standard controllers, e.g. with saturated Proportional, Integral, Derivative (PID) ones.
\end{abstract}

Keywords: Model based control, Model predictive control, Hybrid optimal control, Production plant energy efficiency, Plant energy optimization.

\section{Introduction}

Improving the energy efficiency of manufacturing production systems is nowadays a topic of huge interest. In fact, limiting the $\mathrm{CO} 2$ emissions [1, 2], resizing factory energy supply infrastructures and minimizing plant energy consumptions are leading factors to save plant installation and production costs.

In particular, the reduction of energy consumption is possible through the optimization of the algorithms developed to manage their working function [3,6].

This paper is focused on the definition of the control algorithms for the optimization of the machines' energy efficiency. Specifically, the control policy for a production cell lubrication system has been designed, synthetized and implemented by 
adopting optimal model-based control techniques. These development activities consist in the modeling of the physical process to be controlled, the definition of the constraints on the process variables', and the solution of a suitable optimization problem which has then been implemented into a $\mathrm{C}++$ control platform. A simulation experiment is proposed in the paper to evaluate the resulting control performances.

The paper is organized as follows. Section 2 describes the production cell and the control functional requirements. Section 3 describes the dynamic model of the operating machine lubrication system and details the MPC design, synthesis and implementation. Section 4 shows the results coming from a simulation experiment. Section 5 concludes the paper by summarizing the obtained results and the advantages of the proposed approach, besides hinting to future developments.

\section{Description of the Cell Lubrication System}

The considered production cell is part of the engine assembly line described in [7] whose model is sketched in Fig. 1. The production line machining operation sequence consists of Milling - Welding - Screwing - Drilling activities. However, only the machines $M 1, M 3$ and M4 (for simplicity renumbered in the following as machines $1,2,3$ ) belong to a single production cell because they share some centralized auxiliary systems, such as the fluid lubrication cooling system.

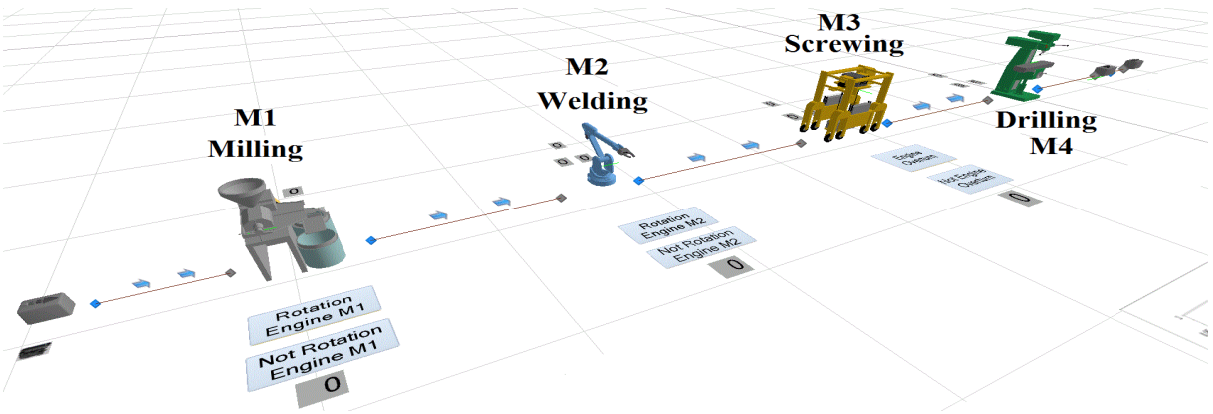

Fig. 1. Engine assembly line layout

During its machining process, each machine produces a specific quantity of heat exchanged with the lubricant fluid, which in turn is cooled from the cooling fluid flowing into the centralized cooling system circuit. As the lubrication fluid temperature grows, the machine control system is able to open an On-Off valve that allows the cooling fluid to flow and thus the lubricant fluid to be cooled.

More specifically, the overall cooling systems operates as follows. Denote by $P_{\text {cool }}$ the thermal power of the centralized cooling system, which can be modulated by the control action, and by $\gamma_{i} \cdot P_{\text {cold }}$ the thermal power exchanged with the $i$-th $(i=1,2,3)$ machine lubrication fluid with temperature $T_{i}$. If all the temperatures $T_{i}, i=1,2,3$, are inside some prescribed limits, the On-Off valve is closed and the no thermal power is provided to the machines. On the contrary, if at least one of these temperatures exceeds an upper bound $T_{\text {hoti }},(i=1,2,3)$, the valve opens and it is set $P_{\text {cold }}=P_{\text {cool }}$. 
The logic behavior of the cooling system can then be defined by the following relations:

$$
\begin{aligned}
& \text { if } \quad \mathrm{V}_{i=1}^{3}\left(T_{i} \geq T_{\text {hoti }}\right) \rightarrow P_{\text {cold }}=P_{\text {cool }} \\
& \text { else } \quad P_{\text {cool }}=0
\end{aligned}
$$

Note however that, due to the different machine characteristics and machining processes, the transferred heat to the lubricant fluids is different from machine to machine, and it is not constant during the machine working cycle.

Table 1 lists the maximum and minimum lubrication fluid temperatures for each machine, referred to a specific machining operation. In addition Table 2 presents the thermal power produced by the considered machining operations. The minimum power produced from a machine is equal to 0 that means that it is not working.

Finally, the room temperature $T_{a}$ is assumed to lie in the range $10-45\left[{ }^{\circ} \mathrm{C}\right]$ while the available cooling power can be modulated between the values $0-3500$ [W].

Table 1. Machine lubrication systems temperature ranges $\left[{ }^{\circ} \mathrm{C}\right]$

\begin{tabular}{r|rrrrrrrr} 
Machines & $\frac{\text { T1min }}{10}$ & $\frac{\text { T1Max }}{140}$ & T2min & & T2Max & T3min & T3Max \\
\hline M2 & & & 10 & 250 & & & \\
M3 & & & & & 10 & 125
\end{tabular}

\begin{tabular}{|c|c|c|c|c|c|c|}
\hline Machines & Plmin & P1Max & $\mathrm{P}_{2 \min }$ & $\mathrm{P}_{2 \mathrm{Max}}$ & P3min & Р3Мax \\
\hline M1 & 0 & 750 & & & & \\
\hline M2 & & & 0 & 500 & & \\
\hline M3 & & & & & 0 & 650 \\
\hline
\end{tabular}

Table 2. Machine lubrication systems thermal power ranges [W]

\section{MPC Design, Synthesis and Implementation}

The control problem related to the centralized lubrication fluid cooling system consists of maintaining the three lubrication fluid temperatures in each specific range by minimizing the energy consumption of the centralized cooling system.

Standard control systems based on industrial controllers are able to manage each lubrication fluid temperature, but they cannot minimize the overall cooling system energy consumption because, due to the resulting decentralized architecture, each local controller only knows the process under its control.

A more suitable technique to solve this class of control problems is Model Predictive Control, or MPC. Nowadays, MPC represents the most widely used advanced control method in the process industry. This is due to the possibility of reformulating the control problem as an optimization one, where different and possibly conflicting goals can be taken into account, as well as to explicitly include constraints on the input and output variables. Moreover, MPC allows for the design of multivariable regulators for large scale systems, with tens or hundreds of variables, see [8]. Thanks to these unique advantages, MPC-based solutions are widely popular in many 
industrial fields characterized by continuous processes, such as the chemical, petrochemical, pulp and paper industries, power networks control and related energy market, see e.g. $[9,10,11]$.

\subsection{MPC Design}

The first step for the application of MPC consists of the modelling of the process to be controlled. In view of the previous considerations, each lubrication fluid receives heat from the machining operation and transfers this energy to the cooling fluid. Therefore, the dynamics of the temperature of the $i$-th machine can be described by the energy balance equation (2), where $\tau$ denotes the continuous time index:

$$
\frac{d T_{i}(\tau)}{d \tau}=\gamma_{i} \cdot\left(T_{a}-T_{i}(\tau)\right)+k_{h i} \cdot P_{h o t i}(\tau)-k_{c i} \cdot P_{c o l d}(\tau)
$$

where

$\gamma_{i} \quad$ is the convection coefficient that models the thermal coupling between lubrication fluid and the external environment at temperature $T_{a}$;

$k_{h i} \quad$ is the coefficient modelling the heat exchanging between the machine and the lubrication fluid;

$k_{c i} \quad$ is the coefficient modelling the heat exchanging between the cooling fluid and the lubrication one.

MPC techniques rely on discrete-time models. Therefore, by exploiting the simple Forward Euler discretization, and denoting by $\Delta \tau$ the adopted sampling time, the discretized form of (2) is:

$$
\begin{aligned}
& \mathrm{T}_{\mathrm{i}}(\mathrm{k} \Delta \tau+\Delta \tau)=\mathrm{T}_{\mathrm{i}}(\mathrm{k} \Delta \tau)+\Delta \tau\left[\gamma_{\mathrm{i}} \cdot\left(\mathrm{T}_{\mathrm{a}}-\mathrm{T}_{\mathrm{i}}(\mathrm{k} \tau)\right)+\mathrm{k}_{\mathrm{hi}} \cdot \mathrm{P}_{\mathrm{hoti}}(\mathrm{k} \tau)-\mathrm{k}_{\mathrm{ci}}\right. \\
& \left.\mathrm{P}_{\text {cold }}(\mathrm{k} \tau)\right], \quad k=0,1,2, \ldots
\end{aligned}
$$

or, denoting by $t$ the discrete-time index,

$$
T_{i}(t+1)=T_{i}(t)+\Delta \tau \cdot\left[\gamma_{i} \cdot\left(T_{a}-T_{i}(t)\right)+k_{h i} \cdot P_{h o t i}(\mathrm{t})-k_{c i} \cdot P_{\text {cold }}(t)\right]
$$

Equations (1), (4) represent the set of dynamic equations and logic statements according to which the MPC can be synthetized.

\subsection{MPC Synthesis}

The MPC synthesis is carried out by implementing the hybrid model defining the discretized physical process (4) and the control logic propositions (1), by means of the HYSDEL software tool [12] which translates the corresponding high level structured language into a Mixed Logic Dynamical (MLD) system formalism [13] of the form (5):

$$
\left\{\begin{array}{c}
x(t+1)=A x(t)+B_{u} u(t)+B_{\delta} \delta(t)+B_{z} z(t) \\
y(t)=C x(t)+D_{u} u(t)+D_{\delta} \delta(t)+D_{z} z(t) \\
E_{\delta} \delta(t)+E_{z} z(t) \leq E_{u} u(t)+E_{x} x(t)+E_{c}
\end{array}\right.
$$


where $x$ is the state variable representing the temperatures $T_{i}, u$ is the control variable representing the cooling power $P_{\text {cool }}, y$ is the output corresponding to the measured state $x$, while $\delta$ and $z$ are vectors of logical and continuous auxiliary variables, respectively.

As previously discussed, the optimization problem is stated to minimize at any sampling time the peak of the overall cooling power $P_{\text {cool }}$ along a future prediction horizon specified by the positive integer $N$. Therefore, the adopted performance index $J$ is:

$$
J=\min _{k=t, \ldots, t+N-1} P_{\text {cool }}(k)
$$

In order to fulfil the required bounds on the lubricant fluid temperatures, the optimization problem is subject to the following constraints:

$$
\begin{aligned}
& T_{1, \text { min }} \leq T_{1}(t) \leq T_{1, \text { max }} \\
& T_{2, \text { min }} \leq T_{2}(t) \leq T_{2, \text { max }} \\
& T_{3, \text { min }} \leq T_{3}(t) \leq T_{3, \max } \\
& P_{c, \text { min }} \leq P_{\text {cool }}(t) \leq P_{c, \text { max }}
\end{aligned}
$$

At any sampling time $t$, once the optimal sequence $P_{\text {cool }}^{o}(k), k=t, \ldots, t+N-1$ of future control variables has been computed, only its first element, i.e. $P_{c o o l}^{o}(t)$, is effectively applied. Then, according to a moving horizon strategy, at the new sampling time $t+1$ the overall procedure is repeated.

It is important to note that the optimization problem above stated may be infeasible due to the nature of the problem under study. In fact, since the cooling system is shared among different machines, it may not be possible to fulfil all the constraints at the same time. For instance, if only one machine is operating and its lubricant has to be cooled, then the others will be cooled as well, by possibly violating their lower bounds. In order to prevent infeasibility of the control solution, it is common practice to transform the hard constraints into soft constraints, in order for them to be violated only if infeasibility occurs. This feature is achieved by heavily weighing these violations in the cost function to be minimized.

In order to complete the MPC synthesis, the heating powers $P_{h o t i}$ have been considered as disturbances acting on the processes. Two solutions are commonly exploited in order to take these disturbances into account, depending on the availability of their future behavior:

a) Prediction available: whenever the future value of the disturbances is available, it should be included into the optimization problem in order to achieve the optimal solution;

b) Prediction not available: if it is not available, it is common practice to assume that the disturbances are constant over the whole prediction horizon, and in particular equal to the current value.

In this work the production cycles have been assumed to be known, then the solution a) has been considered. 


\subsection{MPC Implementation}

The application of the MPC law to a real lubrication system or to an equivalent dynamic simulation model, requires to run the algorithm into a control platform. Then a customized control environment based on the $\mathrm{C}++$ object oriented programming language has been used, by implementing the MPC according the steps showed in Fig. 2:

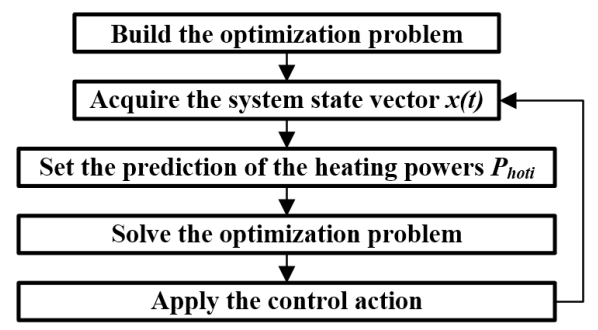

Fig. 2. MPC implementation into the $\mathrm{C}++$ customized control environment

\section{Simulation Results}

Since it has not been possible to apply the developed control system to the real production cell, some simulation experiments have been carried out by running the MLD model of the lubrication system in the MATLAB platform [14] and the MPC in the $\mathrm{C}++$ customized control platform, by interfacing them by means of synchronizing signals. The constraints on the lubricant fluid temperatures have been set accordingly to Table 1. In addition, soft constraints on upper bounds of the temperatures have been used to prevent infeasibility, as previously discussed. The must-be-cooled temperatures $T_{\text {hoti }}$ have been set equal to 100,110 and $85\left[{ }^{\circ} \mathrm{C}\right]$ respectively for $M 1, M 3$ and $M 4$ while the ambient temperature to $25\left[{ }^{\circ} \mathrm{C}\right]$ constant. Finally, the cooling power range is $0-3500$ [W]. The left plots in Fig. 3 depict the selected heating power profiles, corresponding to the working cycles of $M 1, M 3$ and $M 4$, from top to bottom. The first three plots on the right in Fig. 3 show the lubricant temperatures (in solid blue) together with their bounds (in dashed red) and the must-be-cooled temperatures (in solid-dotted magenta). It can be noted that the lower bounds are never violated because they are set as hard constraints. On the other hand, the soft upper bounds are occasionally crossed. As expected, the violations only occur when at least one temperature is close to the lower bound, which cannot be crossed, leading to the upper one crossing (e.g. see the plots at time 7-10s). The bottom-most plot on the right presents the cooling power which always lies inside the given range. 

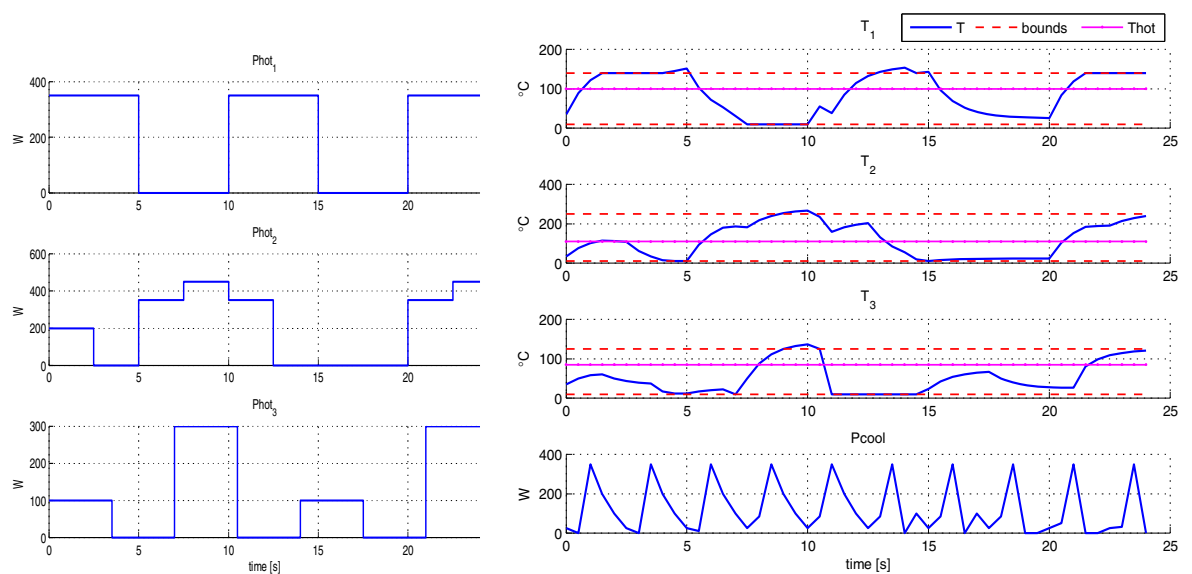

Fig. 3. Simulation experiment results

\section{Concluding Remarks}

In this paper, a production cell based on three operating machines has been considered. In particular, the centralized lubrication cooling system energy management has represented the case study for the design, synthesis and implementation of a model predictive controller. The simulation experiment that has been carried out shows the ability of the MPC to cope with the hybrid control problem in which continuous process variables and corresponding logic constraints have been taken into account. The control action is computed using the a-priori knowledge of the bounds, resulting in better temperature profiles then those obtained with standard controllers. Moreover the small system dimensions require a low computational demand, which leads to fast computing time.

Future works should firstly focus on the deployment of the controller in a real production cell in order to compare the simulation experiment results with the real data acquired from the field. Secondly, a more detailed cooling system modelling would allow for more accurate MPC law calculation and a more realistic control action definition. Finally, an explicit formulation of the MPC, which is currently under study, will allow to replace the on-line optimization with an equivalent, and much lighter, piecewise affine controller, see [15].

Acknowledgements. The research leading to these results has received funding from the European Union's Seventh Framework Programme (FP7/2007-2013) under grant agreement $n^{\circ} 285363$ (EMC2-Factory Eco Manufactured transportation means from Clean and Competitive Factory). 


\section{References}

1. European Commission: ICT and energy efficiency - the case for manufacturing, Recommendations of the Consultation Group, European Commission (February 2009) ISBN: 978-92-79-11306-2

2. Organization for Economic Cooperation and Development (OECED), OECD Key Environmental Indicators (2004),

http: / /www. oecd.org/dataoecd/32/20/31558547.pdf

3. Fysikopoulos, A., Pastras, G., Alexopoulos, T., Chryssolouris, G.: On a generalized approach to manufacturing energy efficiency. The International Journal of Advanced Manufacturing Technology (May 2014)

4. Eberspächer, P., Verla, A.: Realizing energy reduction of machine tools through a control integrated consumption graph-based optimization method. In: 46th CIRP Conference on Manufacturing Systems. Procedia CIRP, vol. 7, pp. 640-645 (2013)

5. Calvanese, M.L., Albertelli, P., Matta, A., Taisch, M.: Analysis of energy consumption in CNC machining centers and determination of optimal cutting conditions. In: Proceedings of the 20th CIRP LCE (2013)

6. Frigerio, N., Matta, A.: Machine Control Policies for Energy Saving in Manufacturing. In: 2013 IEEE International Conference on Automation Science and Engineering (CASE), Madison, WI, August 17-20, pp. 651-656 (2013)

7. Cataldo, A., Taisch, M., Stahl, B.: Modeling, simulation and evaluation of Energy consumption for a manufacturing production line. In: 39th Annual Conference of IEEE on Industrial Electronics Society, IECON 2013, Vienna, Austria, November 10-13 (2013)

8. Camacho, E., Bordons, C.: Model Predictive Control. Springer (2007)

9. Bauer, M., Craig, I.K.: Economic assessment of advanced process control - a survey and framework. J. of Process Control 18, 2-18 (2008)

10. Bendtsen, J., Trangbaek, K., Stoustrup, J.: Hierarchical Model Predictive Control for Resource Distribution. In: 49th IEEE Conf. on Decision and Control, Hilton Atlanta Hotel, Atlanta, GA, USA (2010)

11. Negenborn, R.R., Beccuti, A.G., Demiray, T., Leirens, S., Damm, G., De Schutter, B., Morari, M.: Supervisory hybrid model predictive control for voltage stability of power networks. In: American Control Conference, ACC 2007, New York City, USA, July 9-13, pp. 5444-5449 (2007)

12. Torrisi, F.D., Bemporad, A.: HYSDEL-A Tool for Generating Computational Hybrid Models for Analysis and Synthesis Problems. IEEE Transactions on Control Systems Technology 12(2) (March 2004)

13. Bemporad, A., Morari, M.: Control of systems integrating logic, dynamics, and constraints. Automatica 35, 407-427 (1999)

14. MATLAB, MathWorks, http://www . mathworks. com

15. Bemporad, A., Borrelli, F., Morari, M.: Piecewise linear optimal controllers for hybrid systems. In: Proc. American Control Conference, pp. 1190-1194 (2000) 